Pure and Applied Mathematics Quarterly

Volume 4, Number 2

(Special Issue: In honor of

Fedor Bogomolov, Part 1 of 2)

363 - 381, 2008

\title{
Local Holomorphic Euler Characteristic and Instanton Decay
}

E. Gasparim, T. Köppe and P. Majumdar

\begin{abstract}
We study the local holomorphic Euler characteristic $\chi(x, \mathcal{F})$ of sheaves near a surface singularity obtained from contracting a line $\ell$ inside a smooth surface $Z$. We prove non-existence of sheaves with certain prescribed numerical invariants. Non-existence of instantons on $Z$ with certain charges follows, and we conclude that $\ell^{2}$ poses an obstruction to instanton decay. A Macaulay 2 algorithm to compute $\chi$ is made available at http://www.maths.ed.ac.uk/ s0571100/Instanton/.
\end{abstract}

\section{INTRODUCTION}

Let $\sigma:(\widetilde{X}, D) \rightarrow(X, x)$ be a resolution of an isolated quotient singularity. Let $\widetilde{\mathcal{F}}$ be a reflexive sheaf on $\widetilde{X}$, set $\mathcal{F}:=\left(\sigma_{*} \widetilde{\mathcal{F}}\right)^{\vee}$; notice that there is an embedding $\sigma_{*} \widetilde{\mathcal{F}} \hookrightarrow \mathcal{F}$. Then the local holomorphic Euler characteristic of $\widetilde{\mathcal{F}}$ at $x$ is defined by

$(1.1) \chi(x, \widetilde{\mathcal{F}}):=\chi((\widetilde{X}, D), \widetilde{\mathcal{F}}):=h^{0}\left(X ; \mathcal{F} / \sigma_{*} \widetilde{\mathcal{F}}\right)+\sum_{i=1}^{n-1}(-1)^{i-1} h^{0}\left(X ; R^{i} \sigma_{*} \widetilde{\mathcal{F}}\right)$

For the case when $X$ is an orbifold, Blache [Bl] shows that:

$$
\chi(\widetilde{X}, \widetilde{\mathcal{F}})=\chi(X, \mathcal{F})+\sum_{x \in \operatorname{Sing} X} \chi(x, \widetilde{\mathcal{F}}) .
$$

Received February 27, 2006. 
In this paper we consider rational surface singularities obtained by contracting a line $\ell \cong \mathbb{P}^{1}$ with $\ell^{2}<-1$ inside a smooth surface. To calculate $\chi$ locally, it is enough to study sheaves on a small neighbourhood of the singular point, or on a small neighbourhood of the exceptional set of a resolution. We therefore consider the spaces $Z_{k}:=\operatorname{Tot}\left(\mathcal{O}_{\mathbb{P}^{1}}(-k)\right)$.

We denote by $X_{k}$ the space obtained from $Z_{k}$ by contracting the zero-section $\ell$ to a point, and we let $\pi: Z_{k} \rightarrow X_{k}$ be the contraction map. Since we are interested in applications to instantons, we will consider sheaves $E$ over $Z_{k}$ with $c_{1}(E)=0$. Then $\left.E\right|_{\ell}$ splits by Grothendieck's lemma, and there exists an integer $j \geq 0$ called the splitting type of $E$ such that $\left.E\right|_{\ell} \cong \mathcal{O}(j) \oplus \mathcal{O}(-j)$. Set $Z_{k}^{o}:=Z_{k}-\ell$. We make two simple observations about reflexive sheaves on $Z_{k}$.

Proposition 6.7. Let $E$ be a rank-2 reflexive sheaf on $Z_{k}$ with splitting type $\geq k$, then $\chi(x, E) \geq k-1$.

Proposition 4.1. Let $E_{1}$ and $E_{2}$ be $\mathfrak{s l}(2, \mathbb{C})$-bundles over $Z_{k}$ with splitting types $j_{1}$ and $j_{2}$, respectively. There exists an isomorphism $\left.\left.E_{1}\right|_{Z_{k}^{o}} \cong E_{2}\right|_{Z_{k}^{o}}$ if and only if $j_{1} \equiv j_{2} \bmod k$. In particular, $E_{1}$ can decay totally over $Z_{k}$ if and only if $j_{1} \equiv 0$ $\bmod k$.

This paper consists of applications of the local holomorphic Euler characteristic to problems of existence and decay of instantons. We also discuss the KobayashiHitchin correspondence over $Z_{k}$. We obtain, via discussion of the physical consequences and an ad hoc definition of stability (Definition 5.2), the following conclusions:

Proposition 5.4. There is a one-to-one correspondence between framed $S U(2)$ instantons on $Z_{k}$ with local charge $n$ and framed-stable $\mathfrak{s l}(2, \mathbb{C})$-bundles on $Z_{k}$ with $\chi^{\text {loc }}=n$.

Corollary 5.5. An $\mathfrak{s l}(2, \mathbb{C})$-bundle over $Z_{k}$ represents an instanton if and only if its splitting type is a multiple of $k$.

Theorem 6.8. The minimal local charge of a nontrivial $S U(2)$-instanton on $Z_{k}$ is $\chi_{k}^{\min }=k-1$. The local moduli space of (unframed) instantons on $Z_{k}$ having fixed local charge $\chi_{k}^{\min }$ has dimension $k-2$. 
Acknowledgements: We are honoured to contribute to this volume commemorating Fedya's $60^{\text {th }}$ birthday and are grateful to Ludmil Katzarkov for giving us this opportunity.

The first author thanks the hospitality and generous support of the Mathematics Institute of the Universität Münster, where part of this work was carried out. We thank M. Stillman for assistance with Macaulay 2.

\section{ELEMENTARY BACKGROUND ON INSTANTONS}

Given a principal $S U(2)$-bundle $P \rightarrow X$ over a Riemannian 4-manifold $X$ with $c_{2}(P)=n>0$, an $S U(2)$-instanton of charge $n$ on $X$ is a connection $A$ on $P$ minimising the the Yang-Mills functional

$$
S_{\mathrm{YM}}(A):=\int_{X}\left\|F_{A}\right\|^{2}
$$

where $F_{A}$ is the curvature of the connection $A$. The Yang-Mills equations are the Euler-Lagrange equations corresponding to the functional $S_{\mathrm{YM}}$.

Being non-linear and of second order, the Yang-Mills equations are quite difficult to study. Luckily a linearisation can be obtained easily as follows: The Yang-Mills equation of motion is $D(A) \wedge F(A)=0$. But since the Jacobi identity $D(A) \wedge * F(A)=0$ always holds, any $A$ satisfying $* F_{A}= \pm F_{A}$ solves the Yang-Mills equations of motion. A connection $A$ whose curvature satisfies

$$
* F_{A}=-F_{A},
$$

is called anti-self-dual. Hence, anti-self-dual connections minimise the Yang-Mills functional. For this reason the ASD equations may be seen as a "linear version" of the Yang-Mills equations. Subsequently, from the mathematical point of view, instantons have become synonymous to anti-self-dual connections.

Over a compact Kähler surface $X$, the Kobayashi-Hitchin correspondence (see [LT]) provides an interpretation of irreducible $S U(2)$-instantons of charge $n$ as stable holomorphic $S L(2)$-bundles over $X$ with second Chern class $c_{2}=n$ :

$$
\begin{gathered}
\left\{\begin{array}{c}
\text { irreducible } S U(2) \text {-instantons } \\
\text { of charge } n
\end{array}\right\} \stackrel{\text { K.-H. }}{\Longleftrightarrow}\left\{\begin{array}{c}
\text { stable } S L(2) \text {-bundles } \\
\text { with } c_{2}=n
\end{array}\right\}, \\
\nabla=\bar{\partial}+\partial \longleftrightarrow \bar{\partial} .
\end{gathered}
$$


In particular, when $X$ is a ruled surface, the informal interpretation of instantons as weighted, point-like configurations of concentrated charge has a precise interpretation in terms of jumping lines: An instanton bundle on a ruled surface has a generic splitting $\mathcal{O}(a) \oplus \mathcal{O}(-a)$, which is the same for all but finitely many lines of the ruling, called the jumping lines. In this interpretation, the weight of the points in the configuration corresponds to the multiplicity of the jumps, and the topological charge (= the second Chern class) is given as the sum of all multiplicities.

Multiplicities of jumps on lines having $\ell^{2}=0$, i.e. on trivial families $\mathbb{C} P^{1} \times \mathbb{C}$, are quite well understood (cf. $[\mathrm{BHMM}],[\mathrm{HM}]$ ). Here we study the behaviour of instantons around lines with negative self-intersection $\ell^{2}=-k$. The case $k=1$ corresponds to the blow-up of a surface at a point and was studied in several papers, e.g. [GO], [Ga1], [Kn]. Near a $-k$-line, with $-k<0$, an instanton has two independent local numerical invariants: the height and the width (see Definition 2.3), whose sum gives the multiplicity or local charge. The label "local charge" comes from the translation into algebraic geometry via the Kobayashi-Hitchin correspondence:

In the first case, let $\widetilde{X}$ be a compact complex surface containing a -1-line, and let $\pi: \widetilde{X} \rightarrow X$ be the blow-down of $\ell$ to a point $x_{0} \in X$. If $E$ is a bundle on $\widetilde{X}$, then the local second Chern class of $E$ near $\ell$ is by definition

$$
c_{2}^{\mathrm{loc}}(\ell, E):=c_{2}(E)-c_{2}\left(\left(\pi_{*} E\right)^{\vee \vee}\right) .
$$

Thus, a local version of the Kobayashi-Hitchin correspondence justifies the terminology

$$
\left\{\begin{array}{c}
\text { local charge of an } \\
\text { instanton }
\end{array}\right\} \stackrel{\mathrm{K} \cdot-\mathrm{H}}{\Longleftrightarrow}\left\{\text { local } c_{2} \text { of a bundle }\right\} .
$$

Still near the -1-line, an application of Hirzebruch-Riemann-Roch gives

$$
c_{2}^{\text {loc }}(\ell, E)=h^{0}\left(X ;\left(\pi_{*} E\right)^{\vee \vee} / \pi_{*} E\right)+h^{0}\left(X ; R^{1} \pi_{*} E\right),
$$

which by (1.1) is the local holomorphic Euler characteristic of $E$ near $\ell$.

However, the analogue of (2.2) for a bundle near a $-k$-line, where $k \geq 2$, is a more complicated issue, simply because contracting such a line produces a singularity; and there exist at least three nonequivalent definitions of Chern classes for singular varieties. To avoid carrying this problem over to instantons, it 
is more convenient to simply consider the local holomorphic Euler characteristic, and set:

Definition 2.3. Let $E$ be an instanton bundle over a smooth surface $Z$ containing a $-k$ line $\ell$ and let $\pi: Z \rightarrow X$ be map that contracts of $\ell$ to a point. The local charge of $E$ around $\ell$ is:

$$
\chi^{\mathrm{loc}}(E)=\chi(\ell, E):=h^{0}\left(X ;\left(\pi_{*} E\right)^{\vee \vee} / \pi_{*} E\right)+h^{0}\left(X ; R^{1} \pi_{*} E\right) .
$$

The right hand side contains two independent holomorphic invariants:

$\mathbf{w}(E):=h^{0}\left(X ;\left(\pi_{*} E\right)^{\vee \vee} / \pi_{*} E\right)$ is called the width of the instanton and measures how far the direct image is from being a bundle;

$\mathbf{h}(E):=h^{0}\left(X ; R^{1} \pi_{*} E\right)$ is called the height of the instanton and measures how far the bundle is from being a split extension.

From equation $(1.2), \chi(E)=\chi^{\mathrm{loc}}(E)+\chi\left(\left(\pi_{*} E\right)^{\vee \vee}\right)$, so we can say that the local charge measures the loss of total charge suffered by contracting $\ell$.

\section{Holomorphic SURGERY AND INSTANTON DECAY}

We first describe informally the ideas behind holomorphic surgery and decay and then give the precise definitions. A decay of an instanton is a transformation that lowers the total charge; a local decay around $\ell$ is a transformation that keeps the instanton fixed outside $\ell$ but lowers the local charge near $\ell$, and consequently lowers the global charge as well.

When an instanton is represented by a holomorphic bundle $E$, then holomorphic surgery provides a precise way to obtain local decay: If the surface $Z$ contains a line $\ell$ and $N=N(\ell)$ is a small neighbourhood of $\ell$ in the analytic topology, then lowering the charge of $E$ around $\ell$ means to replace $\left.E\right|_{N}$ by some $\left.E^{\prime}\right|_{N}$ with smaller local charge, while keeping $E$ fixed on $Z-\ell$. The outcome is a new holomorphic bundle which is isomorphic to $E$ over $Z-\ell$, but which has smaller $c_{2}$.

Remark 3.1. Note that holomorphic surgery takes one instanton bundle to another instanton bundle; that is, the surgery process keeps $c_{1}=0$, and consequently differs from the more familiar process of elementary transformations (which does not move between instantons). 
Definition 3.2. Two instanton bundles $E_{1}$ and $E_{2}$ on $X$ that are related by holomorphic surgery around $\ell$ must satisfy $\left.\left.E_{1}\right|_{X-\ell} \cong E_{2}\right|_{X-\ell}$ holomorphically. If in addition $c_{2}\left(E_{1}\right)>c_{2}\left(E_{2}\right)$, we will say that $E_{1}$ decays to $E_{2}$.

We fix a compact surface $Z$ containing a line $\ell$ with $\ell^{2}=-k$, as in the introduction, with $\pi: Z \rightarrow X$ the contraction of $\ell$, and a decomposition $Z=(Z-\ell) \cup N(\ell)$. We will see in Proposition 5.3 that instantons correspond to bundles that are trivial on $Z^{o}:=N(\ell)-\ell=(Z-\ell) \cap N(\ell)$. So, instanton bundles can be given frames on $Z^{\circ}$. We will use theses frames (see definition 3.3 below) in our constructions. Note that $\left(Z_{k}, Z_{k}^{o}\right)$ describes the local situation, whereas $\left(Z, Z^{o}\right)$ describes the global situation on a compact manifold. (Although it is not true that any 2-dimensional tubular neighbourhood of a $-k$-line $\ell$ is biholomorphic to $Z_{k}$, it is a consequence of [Ga2] that holomorphic bundles on both $N(\ell)$ and $Z_{k}$ are completely determined by a finite infinitesimal neighbourhood of $\ell$, so that for the purposes of holomorphic surgery and instanton decay, $N(\ell)$ and $Z_{k}$ can be identified.)

Definition 3.3. Let $\pi_{F}: F \rightarrow Z$ be a bundle over $Z$ that is trivial over $Z^{o}:=Z-$ $Y$. Given two pairs $f=\left(f_{1}, f_{2}\right): Z^{o} \rightarrow \pi_{F}^{-1}\left(Z^{o}\right)$ and $g=\left(g_{1}, g_{2}\right): Z^{o} \rightarrow \pi_{F}^{-1}\left(Z^{o}\right)$ of fibrewise linearly independent holomorphic sections of $\left.F\right|_{Z^{\circ}}$, we say that $f$ is equivalent to $g$ (written $f \sim g$ ) if $\phi:=g \circ f^{-1}:\left.\left.V\right|_{Z^{\circ}} \rightarrow V\right|_{Z^{\circ}}$ extends to a holomorphic map $\phi: F \rightarrow F$ over the entire $Z$. A frame of $F$ over $Z^{o}$ is an equivalence class of fibrewise linearly independent holomorphic sections of $F$ over $Z^{\circ}$.

- A framed bundle $\bar{E}^{f}$ on $Z$ is a pair consisting of a bundle $\pi_{\bar{E}}$ : $\bar{E} \rightarrow Z$ together with a frame of $\bar{E}$ over $Z^{o}:=N(\ell)-\ell$.

- A framed bundle $V^{f}$ on $Z_{k}:=\operatorname{Tot}\left(\mathcal{O}_{\mathbb{P}^{1}}(-k)\right)$ is a pair consisting of a bundle $\pi_{V}: V \rightarrow Z_{k}$ together with a frame of $V$ over $Z_{k}^{o}$.

- A framed bundle $E^{f}$ on $X$ is a pair consisting of a bundle $\pi_{E}: E \rightarrow X$ together with a frame of $E$ over $N(x)-\{x\}$, where $N(x)$ is a small disk neighbourhood of $x$. We will always take $N(x):=\pi(N(\ell))$.

Proposition 3.4. An isomorphism class $\left[\bar{E}^{f}\right]$ of a framed bundle on $Z$ is uniquely determined by a pair of isomorphism classes of framed bundles $\left[E^{f}\right]$ on $X$ and $\left[V^{f}\right]$ on $Z_{k}$. We write $\bar{E}^{f}=\left(E^{f}, V^{f}\right)$. 
Proof. One needs to observe that any reflexive sheaf on $X$ is completely determined by the complement of the point $x$, cf. [Ha2, Prop. 1.6]. Hence $E=\left(\pi_{*} \bar{E}\right)^{\vee \vee}$ is trivial on $N(x)$ and is completely determined by $\left.E\right|_{X-\{x\}}$. The rest of the proof is just a verification that the framings have been conveniently defined.

The contraction map gives an isomorphism $i_{1}: Z-\ell \rightarrow X-\{x\}$. Based on [Ga2] we may assume that there is an isomorphism $i_{2}: N(\ell) \rightarrow Z_{k}$. These induce isomorphisms on the deleted neighbourhoods

$$
N(x)-\{x\} \stackrel{i_{1}}{\longleftarrow} Z^{o} \stackrel{i_{2}}{\longrightarrow} Z_{k}^{o} .
$$

By construction, $\bar{E}=E \sqcup_{\left(s_{1}, s_{2}\right)=\left(t_{1}, t_{2}\right)} V$ is made by identifying the bundles as well as the sections over $Z^{o}$, so that the bundles satisfy $\left.\bar{E}\right|_{Z^{o}}=i_{1}^{*}\left(\left.E\right|_{N(x)-\{x\}}\right)=$ $i_{2}^{*}\left(\left.V\right|_{Z_{k}^{o}}\right)$, and the framing $\left(f_{1}, f_{2}\right)$ of $\bar{E}$ satisfies $\left(f_{1}, f_{2}\right)=\left(s_{1}, s_{2}\right) \circ i_{1}=\left(t_{1}, t_{2}\right) \circ i_{2}$.

Let $\phi: E^{f} \rightarrow E^{\prime f}$ be an isomorphism such that $\phi \circ\left(s_{1}, s_{2}\right)=\left(s_{1}^{\prime}, s_{2}^{\prime}\right)$, and let $\xi: V^{f} \rightarrow V^{\prime f}$ be an isomorphism such that $\xi \circ\left(t_{1}, t_{2}\right)=\left(t_{1}^{\prime}, t_{2}^{\prime}\right)$. We have the following diagram of bundle maps:

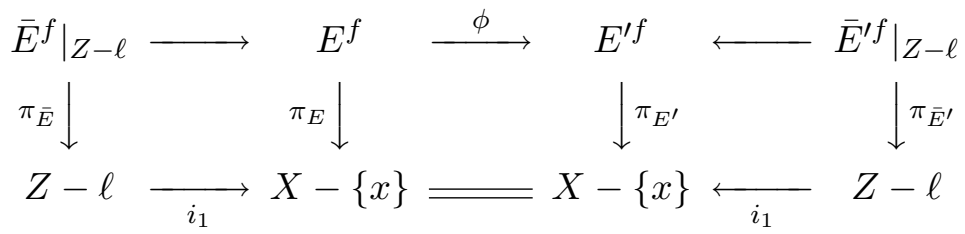

Hence,

$$
\left.\bar{E}^{\prime f}\right|_{Z-\ell}=i_{1}^{*}\left(\left.E^{\prime f}\right|_{X-\{x\}}\right)=i_{1}^{*} \circ \phi\left(\left.E^{f}\right|_{X-\{x\}}\right)=i_{1}^{*} \circ \phi \circ i_{1 *}\left(\left.\bar{E}^{f}\right|_{Z-\ell}\right),
$$

showing that $i_{1}^{*} \circ \phi \circ i_{1 *}$ is an isomorphism of $\bar{E}$ and $\bar{E}^{\prime}$ over $Z-\ell$ such that

$$
\phi \circ\left(f_{1}, f_{2}\right)=\phi \circ\left(s_{1}, s_{2}\right) \circ i_{1}=\left(s_{1}^{\prime}, s_{2}^{\prime}\right) \circ i_{1}=\left(f_{1}^{\prime}, f_{2}^{\prime}\right) .
$$

On the other hand we have a second diagram of bundle maps:

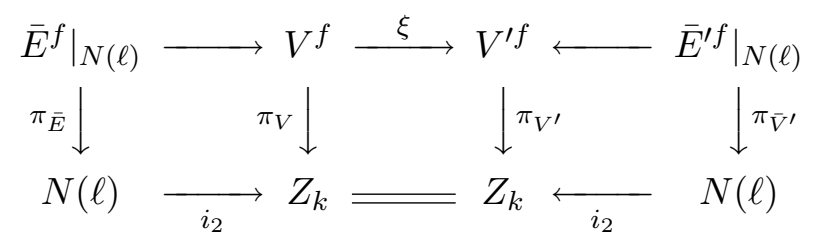

Therefore,

$$
\left.\bar{E}^{\prime f}\right|_{N(\ell)}=i_{2}^{*}\left(\left.V^{\prime f}\right|_{Z_{k}}\right)=i_{2}^{*} \circ \xi\left(\left.V^{f}\right|_{Z_{k}}\right)=i_{2}^{*} \circ \xi \circ i_{2 *}\left(\left.\bar{E}^{f}\right|_{N(\ell)}\right),
$$


showing that $i_{2}^{*} \circ \xi \circ i_{2 *}$ is an isomorphism of $\bar{E}$ and $\bar{E}^{\prime}$ over $N(\ell)$ such that

$$
\xi \circ\left(f_{1}, f_{2}\right)=\xi \circ\left(t_{1}, t_{2}\right) \circ i_{2}=\left(t_{1}^{\prime}, t_{2}^{\prime}\right) \circ i_{2}=\left(f_{1}^{\prime}, f_{2}^{\prime}\right) .
$$

These isomorphisms agree over the intersection $Z^{\circ}$. In fact, by (3.5) and (3.7),

$$
\begin{aligned}
i_{1}^{*} \circ \phi \circ i_{1 *}\left(\left.\bar{E}^{f}\right|_{Z^{o}}\right) & =i_{1}^{*} \circ \phi\left(\left.E^{f}\right|_{N(x)}\right)=i_{1}^{*}\left(\left.E^{\prime f}\right|_{N(x)}\right) \\
= & i_{2}^{*}\left(\left.V^{\prime f}\right|_{Z_{k}-\{0\}}\right)=i_{2}^{*} \circ \xi\left(\left.V^{f}\right|_{Z_{k}-\{0\}}\right)=i_{2}^{*} \circ \xi \circ i_{2 *}\left(\left.\bar{E}^{f}\right|_{Z^{o}}\right),
\end{aligned}
$$

and moreover they also preserve the framings over the intersection, since over $Z^{o}$ we have, by (3.6) and (3.8),

$$
\phi \circ\left(f_{1}, f_{2}\right)=\left(f_{1}^{\prime}, f_{2}^{\prime}\right)=\xi \circ\left(f_{1}, f_{2}\right) .
$$

By the gluing lemma this gives an isomorphism over the entire space $\bar{X}$, and we get $\bar{E}^{\prime} \simeq \bar{E}$.

Note: Here we have only defined surgery for framed bundles on surfaces. A similar definition of holomorphic surgery can be given in much greater generality for decorated bundles on higher-dimensional varieties; for instance, a broader sense of framing can be used by fixing the isomorphism type of the bundles on a subvariety.

Using (2.1) we can re-state Proposition 3.4 in terms of instantons:

Proposition 3.9. If $\nabla$ and $\nabla^{\prime}$ are instantons on $Z$, with $\nabla^{\prime}$ obtained from $\nabla$ by local decay, then

$$
\text { global charge }\left(\nabla^{\prime}\right)=\text { global charge }(\nabla)-\text { local charge }(\nabla, \ell) .
$$

Proof. Just combine Proposition 3.4 and equality (1.2).

\section{When is total DeCAy NeAR $\ell$ POSSiBle?}

Consider the questions: Can every bundle decay totally around $\ell$, that is, is every bundle related by holomorphic surgery to a bundle that is trivial around $\ell$ ? Can decay by 1 always happen, that is can every charge $n$ instanton decay locally to charge $n-1$ ? In the case $k=1$ the answers are "yes", but for $k>2$ we will show that the answers to both questions are "no".

In this section we use the well-known concept of elementary transformations of Maruyama [M], which we now recall: Let $E$ be a vector bundle over an algebraic 
variety $W$. Choose a line bundle $L$ over a Cartier divisor $D \subset W$ and a surjection $r: E \rightarrow L$ induced by a surjection $\rho:\left.E\right|_{D} \rightarrow L$. Set $E^{\prime}:=\operatorname{ker}(r)$ and $L^{\prime}:=\operatorname{ker}(\rho)$. Since $D$ is a Cartier divisor, $E^{\prime}$ is a vector bundle on $W$. By definition $E^{\prime}$ is called the vector bundle obtained from $E$ by making the elementary transformation induced by $r$, denoted

$$
E^{\prime}=\operatorname{Elm}_{L}(E) .
$$

The following diagram, called the display of the elementary transformation, clarifies the situation:

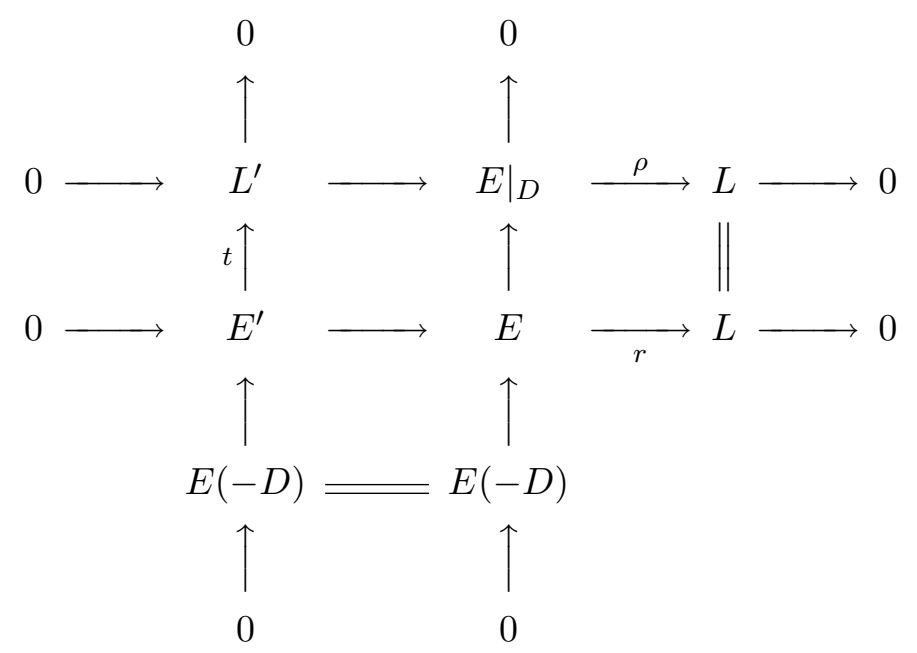

Note that the elementary transformation does not change $E$ outside the divisor, that is, $\left.\left.E\right|_{W-D} \cong E^{\prime}\right|_{W-D}$.

Proposition 4.1. Let $E_{1}$ and $E_{2}$ be $\mathfrak{s l}(2, \mathbb{C})$-bundles over $Z_{k}$ with splitting types $j_{1}$ and $j_{2}$, respectively. There exists an isomorphism $\left.\left.E_{1}\right|_{Z_{k}^{o}} \cong E_{2}\right|_{Z_{k}^{o}}$ if and only if $j_{1} \equiv j_{2} \bmod k$. In particular, $E_{1}$ can decay totally over $Z_{k}$ if and only if $j_{1} \equiv 0$ $\bmod k$.

Proof. We first claim that the bundle $\mathcal{O}_{\ell}(-k)$ is trivial on $Z_{k}^{o}$. In fact, if $u=0$ is the equation of $\ell$, then $s(z, u)=u$ determines a section of $\mathcal{O}_{\ell}(-k)$ that does not vanish on $Z_{k}^{o}$.

If a bundle $E$ over $Z_{k}$ has splitting type $j$, then by definition, $\left.E\right|_{\ell} \cong \mathcal{O}_{\ell}(-j) \oplus$ $\mathcal{O}_{\ell}(j)$. So there is a surjection $\rho:\left.E\right|_{\ell} \rightarrow \mathcal{O}_{\ell}(j)$. The bundle $E^{\prime}=\operatorname{Elm}_{\mathcal{O}_{\ell}(j)}(E)$ splits over $\ell$ as $\mathcal{O}_{\ell}(-j) \oplus \mathcal{O}_{\ell}(j+k)$. Therefore we can use the surjection $\rho:\left.E^{\prime}\right|_{\ell} \rightarrow$ $\mathcal{O}_{\ell}(j+k)$ to perform a second elementary transformation, and we obtain bundle $E^{\prime \prime}=\operatorname{Elm}_{\mathcal{O}_{\ell}(j+k)}\left(E^{\prime}\right)$, which splits over $\ell$ as $\mathcal{O}_{\ell}(-j) \oplus \mathcal{O}_{\ell}(j+2 k)$ and has first 
Chern class $2 k$. Tensoring by $\mathcal{O}_{\ell}(-k)$ we get back to an $\mathfrak{s l}(2, \mathbb{C})$-bundle with splitting type $j+k$. Hence, the transformation

$$
\Phi(E)=\otimes \mathcal{O}(-k) \circ \operatorname{Elm}_{\mathcal{O}_{\ell}(j+k)} \circ \operatorname{Elm}_{\mathcal{O}_{\ell}(j)}(E)
$$

increases the splitting type by $k$ while keeping the isomorphism type of $E$ over $Z_{k}^{o}$. So we need only to analyse bundles with splitting type $j<k$.

If $j=0$, the bundle is globally trivial on $Z_{k}$. If $j \neq 0$, then $\left.E\right|_{Z_{k}^{o}}$ induces a non-zero element on the fundamental group $\pi_{1}\left(Z_{k}^{o}\right)=\mathbb{Z} / k \mathbb{Z}$.

One interesting consequence of Proposition 4.1 is that instantons do not correspond to bundles whose splitting type does not divide $k$. Consequently, using the results of the following two sections, we will deduce:

Corollary 4.2. The self-intersection number of $\ell$ provides an obstruction to the existence of instantons with certain prescribed numerical invariants.

In particular, it is not always possible for the local charge to decay by one unless $k=1$ or 2 .

Corollary 4.3. The self-intersection number of $\ell$ poses an obstruction to instanton decay.

Example 4.4. Here some examples, which will be proved below.

(1) There is no nontrivial instanton with local charge $\leq k-2$ over the space $Z_{k}$ when $k>2$.

(2) For $k \geq 2$, there exist $(k-2)$-dimensional families of (unframed) instantons with local charge $k-1$ over $Z_{k}$.

\section{Existence of instantons}

In [Ga2] it is shown that every holomorphic bundle on $Z_{k}$ is an algebraic extension of line bundles. It then follows that any rank-2 bundle $E$ over $Z_{k}$ with $c_{1}(E)=0$ is an extension of the form

$$
0 \rightarrow \mathcal{O}(-j) \rightarrow E \rightarrow \mathcal{O}(j) \rightarrow 0
$$

Thus existence of moduli $\mathcal{M}_{j}(k)$ of bundles with any splitting type $j$ over $Z_{k}$ is an immediate consequence of the fact that $\operatorname{Ext}^{1}\left(\mathcal{O}_{Z_{k}}(j), \mathcal{O}_{Z_{k}}(-j)\right) \neq \varnothing$. Moreover, 
in [BGK, Theorem 4.2] it is shown that for $j>k$

$$
\operatorname{dim} \mathcal{M}_{j}(k)=2 j-k-2 .
$$

Note that $\operatorname{dim} \mathcal{M}_{j}(k)$ is not the dimension of $\operatorname{Ext}^{1}\left(\mathcal{O}_{Z_{k}}(j), \mathcal{O}_{Z_{k}}(-j)\right)$ as a vector space, since bundle isomorphisms impose several equivalences; rather it is the dimension of the dense, open stratum of $\mathcal{M}_{j}(k)$ seen as a variety. Now we analyse which of these bundles correspond to instantons. Firstly, we look at them from the point of view of decay, and secondly from the point of view of differential geometry.

The energy of an instanton on $X$ is given by $\frac{1}{2 g^{2}} \int_{X}\|F\|^{2}$. The minimum possible energy of the instanton is bounded from below by the total charge of the instanton. To see this, note the following inequalities:

$$
0 \leq \int_{X}\|F \pm * F\|^{2}=2 \int_{X}\|F \wedge * F \pm F \wedge F\|
$$

Therefore,

$$
\frac{1}{2 g^{2}} \int_{X}\|F\|^{2}=\frac{1}{2 g^{2}} \int_{X}\|F \wedge * F\| \geq \frac{1}{2 g^{2}}\left|\int_{X} F \wedge F\right|=\frac{8 \pi^{2}|n|}{g^{2}} .
$$

Consequently, the probability of finding an instanton with $c_{2}=n$ is $\propto e^{-n}$. Arguing from the physical point of view, since systems always tend to go to their lowest energy state, an instanton with a high charge will prefer to decay to an instanton of a lower charge unless there is some obstruction to its decay. It is reasonable to expect that this behaviour holds locally as well. Thus it should be possible to lower a local charge by a local transformation. Instanton bundles, therefore, ought to allow for full local decay; combining with Proposition 4.1 this implies that only bundles which are trivial on $Z_{k}^{o}$ can correspond to instantons. Moreover, the finite-energy condition for instantons, viz. $\int_{X}\|F\|^{2} \leq \infty$, requires that $F \rightarrow 0$ at infinity, and accordingly an instanton bundle $E$ on $Z_{k}$ should be trivial and trivialised at infinity. This requirement in turn fixes boundary conditions and guarantees that instantons have good gluing properties.

Mathematically, the correct way to decide which bundles correspond to instantons is to go through the Kobayashi-Hitchin correspondence (cf. [LT]). A unitary, anti-self-dual connection $\nabla$ on a smooth bundle $E$ decomposes as $\nabla=\partial+\bar{\partial}$, where $\bar{\partial}$ is considered as a holomorphic structure on $E$; and the Kobayashi-Hitchin correspondence claims that the map $\nabla \mapsto \bar{\partial}$ is invertible. In the compact case, this correspondence was proved by Donaldson [D1] for projective algebraic surfaces, 
by Uhlenbeck and Yau [UY] for Kähler surfaces, and by Buchdahl [Bu] for surfaces with a Gauduchon metric. In the non-compact case, this correspondence was proved by Donaldson [D2] for $\mathbb{C}^{2}$ and by King [Kn] for $\mathbb{C}^{2}$ blown up at the origin, which in this paper is denoted by $Z_{1}$. In the former, instantons on $\mathbb{C}^{2}$ are identified with instantons on $\mathbb{C} P^{2}$ framed at a line at infinity; and in the latter framed instantons on $Z_{1}$ are identified with instantons on the first Hirzebruch surface $\Sigma_{1}$ which are trivial on the line at infinity. As in the non-compact cases of $\mathbb{C}^{2}$ and $Z_{1}$ we identify framed instantons on $Z_{k}$ with instantons on the $k^{\text {th }}$ Hirzebruch surface $\Sigma_{k}:=\mathbb{P}\left(\mathcal{O}_{\mathbb{P}^{1}}(k) \oplus \mathcal{O}_{\mathbb{P}^{1}}\right)$ trivialised on the line at infinity.

LeBrun [LB1] provided metrics over the spaces $Z_{k}$ that are well suited for instanton problems. He showed that $Z_{k}$ admits a complete, zero scalar curvature, asymptotically flat Kähler metric $g$. In particular, this metric is anti-self-dual. Moreover, he showed that up to multiplication by an overall constant, there is exactly one such metric $g$ which is $S U(2)$-invariant. Using this asymptotically flat metric, triviality at the line at infinity still seems a natural condition to impose. In fact, consider the orbifold $\bar{Z}_{k}$ obtained from $Z_{k}$ by adding one point at infinity, or equivalently, obtained from $\Sigma_{k}$ by contracting the line at infinity $\ell_{\infty}$. Then it follows from [LB2, p. 235] that $\bar{Z}_{k}$ is an ASD conformal orbifold compactification of $Z_{k}$. This orbifold compactification of $Z_{k}$ has an orbifold twistor space $W$, cf. [LB3]. The complex structure on $Z_{k}$ yields a complex surface in $W$, and adding the orbifold twistor line at infinity compactifies this surface to the Hirzebruch surface $\Sigma_{k}$. Using the Ward correspondence together with Uhlenbeck's removable singularities theorem [U], an $L^{2}$-instanton on $Z_{k}$ gives rise to a holomorphic bundle on $\Sigma_{k}$ that is trivial on $\ell_{\infty}$. So, once again, from this second point of view we arrive at the conclusion that instanton bundles on $Z_{k}$ should be the ones that are trivial at infinity. We set the ad hoc definition of framed stability, cf. Definition 3.3.

Definition 5.2. A rank-2 bundle over $Z_{k}$ is called framed-stable if it is holomorphically trivial and framed on $Z_{k}^{o}$.

This allows a statement the Kobayashi-Hitchin correspondence on $Z_{k}$ :

Proposition 5.3. There exists a one-to-one correspondence between framed $S U(2)$ instantons on $Z_{k}$ with local charge $n$ and framed-stable $\mathfrak{s l}(2, \mathbb{C})$-bundles on $Z_{k}$ with $\chi^{\text {loc }}=n$. 
Schematically,

$$
\left\{\begin{array}{c}
S U(2) \text {-instantons on } \\
Z_{k} \text { with local charge } n
\end{array}\right\} \stackrel{\text { K.-H. }}{\Longleftrightarrow}\left\{\begin{array}{c}
\text { stable } S L(2) \text {-bundles } \\
\text { on } Z_{k} \text { with } \chi^{\text {loc }}=n
\end{array}\right\} .
$$

Corollary 5.5. An $\mathfrak{s l}(2, \mathbb{C})$-bundle over $Z_{k}$ represents an instanton if and only if its splitting type is a multiple of $k$.

Proof. By definition an instanton bundle must be trivial at infinity, now apply Proposition 4.1.

In particular, note that any bundle on $Z_{k}$ with splitting type $j \not \equiv 0 \bmod k$ does not correspond to an instanton; the physical interpretation of such a bundle does not seem to be known.

\section{Gaps in LOCAL Charges AND MOdUli}

In this section we study gaps in local charges. We show that not all numerically admissible values of $\chi$ occur for instanton bundles when $k>2$. Recall that by Definition 2.3 the local charge is the sum of the width and the height: $\chi^{\text {loc }}(E)=$ $\mathbf{w}(E)+\mathbf{h}(E)=h^{0}\left(X ;\left(\pi_{*} E\right)^{\vee \vee} / \pi_{*} E\right)+h^{0}\left(X ; R^{1} \pi_{*} E\right)$.

6.1. Direct computation of instanton widths. Results in this section depend on a number of "direct calculations". We explain briefly how those are carried out and provide an open computer implementation. We outline the computer algorithm, following closely the ideas in $[\mathrm{GaS}]$ and keeping to minimal detail. The implementation of the algorithm can be obtained from

http://www.maths.ed.ac.uk/ s0571100/Instanton/. Our language of choice is Macaulay 2 for its native support of high-level concepts of commutative algebra (such as modules, generators, cokernels); though conceivably a different computer algebra software may be used.

Let $E \rightarrow Z_{k}$ be a holomorphic rank-2 vector bundle over the complex surface $Z_{k}=\operatorname{Tot}(\mathcal{O}(-k))$ with $c_{1}(E)=0$. The canonical coordinates on $Z_{k}=U \cup V$ are $U=\{z, u\}$ and $V=\{\xi, v\}$ such that $\xi=z^{-1}$ and $v=z^{k} u$. A holomorphic bundle $E$ on $Z_{k}$ is algebraic [Ga2]. If the splitting type of $E$ is $j$, then it can be expressed by a canonical transition function

$$
T=\left(\begin{array}{cc}
z^{j} & p \\
0 & z^{-j}
\end{array}\right),
$$


where

$$
p(z, u)=\sum_{r=1}^{\left\lfloor\frac{2 j-2}{k}\right\rfloor} \sum_{s=k i-j+1}^{j-1} p_{r s} u^{r} z^{s} .
$$

The computation of the instanton width is now equivalent to the computation of the dimension of the cokernel of the natural evaluation map $M \hookrightarrow M^{\vee \vee}$, where $M$ is a module that is related to the space of holomorphic sections of $E$ : Let $(a, b)$ be a generic section of $E$ given over the $(z, u)$-chart by functions $a, b \in \mathbb{C}[[z, u]]$. This implies that on the other chart, the local section

$$
T\left(\begin{array}{l}
a \\
b
\end{array}\right)=\left(\begin{array}{c}
z^{j} a+p b \\
z^{-j} b
\end{array}\right)
$$

is holomorphic in $\left(z^{-1}, z^{k} u\right)$. Writing $a(z, u)=a_{r s} u^{r} z^{s}$ and $b(z, u)=b_{r s} u^{r} z^{s}$, this means that for each fixed index $r$ only a finite number of $a_{r s}$ and $b_{r s}$ can be nonzero, and there are relations between the non-zero coefficients of $a$ and $b$ (unless $p \equiv 0)$. The space of sections of $E$ is thus generated by terms $\left(u^{r} z^{s}, u^{r^{\prime}} z^{s^{\prime}}\right)$, of which most are multiples or linear combinations of a finite set of true generators.

For our computation we need to consider generators and relations after contracting the zero-section of $Z_{k}$ to a point, i.e. on the direct image under the contracting map $\pi: Z_{k} \rightarrow X_{k}$. Here $X_{k}$ is the singular surface (smooth only for $k=1)$ given in coordinates by $S=\left\{x_{0}, \ldots, x_{k}\right\} /\left(x_{i} x_{i+t}-x_{i+1} x_{i+t-1}\right)$ with $0 \leq i \leq i+t \leq k+1$, and the map $\pi$ is given by $x_{i}=z^{i} u$. The module $M$ is now the space of sections of $E$ when the relations are expressed in terms of the $x_{i}$ downstairs, i.e. as an $S$-module.

Example 6.2. For the first two values of $k$ we have $Z_{1} \rightarrow X_{1}=\mathbb{C}^{2}=\left\{x_{0}, x_{1}\right\}$ and $Z_{2} \rightarrow X_{2}=\left\{x_{0}, x_{1}, x_{2}\right\} /\left(x_{0} x_{2}-x_{1}^{2}\right)$, where $u \mapsto x_{0}, z u \mapsto x_{1}$ and $z^{2} u \mapsto x_{2}$.

The first two generators coming from $b_{00}$ and $b_{01}$ are $\beta_{0}=(0,1)$ and $\beta_{1}=(0, z)$, respectively, and they are related over $S$ by

$$
\begin{array}{ll}
x_{1} \beta_{0}=x_{0} \beta_{1} & \\
x_{2} \beta_{0}=x_{1} \beta_{1} & \text { (on } \left.Z_{1} \text { and } Z_{2} \text { ), and }\right) .
\end{array}
$$

The concrete case $j=k$ and $p(z, u)=z u$ is worked out in the proof of Theorem 6.8 . 
Computer algorithm. The automatic computation of the instanton width of a bundle $E$ with $c_{1}(E)=0$, splitting type $j$ and extension class $p$ can now proceed in several stages:

(1) (Optional) The extension class $p$ may possibly be reduced to a smaller, cohomologous class $p^{\prime}$ by truncating terms according to (6.1), but care needs to be taken when $u \nmid p$. This step is only useful to optimise computation time, it is not necessary for the algorithm to work.

(2) Define a generic section $(a, b)$ with $a(z, u)=\sum_{r, s} a_{r s} u^{r} z^{s}$ and $b(z, u)=$ $\sum_{r, s} b_{r s} u^{r} z^{s}$. There exist bounds on $r$ above which all generators corresponding to the $a_{r} s$ and $b_{r} s$ terms are guaranteed to be multiples (over $S$ ) of the lower generators, so these are genuine polynomials. Moreover, $b$ can and must be chosen so that $z^{-j} b$ is holomorphic in $z^{-1}$ and $z^{k} u$.

(3) Compute the first coordinate of the section on the second chart: $f=$ $z^{j} a+p u$. Now for each term $z^{s} u^{r}$, whenever $s>k r$ the coefficient must vanish; this gives relations between the coefficients.

(4) The module $M$ is now built up step by step by substituting the relations back into $a$ and $b$, and setting one coefficient to 1 and all others to 0 (call the result $a_{1}, b_{1}$ just for now), transforming $\left(a_{1}, b_{1}\right)$ into expressions over $S$ and adding the resulting vector as a generator of $M$. After doing this for all coefficients, a presentation for $M$ as a module over $S$ is obtained. (See [GaS] for details of this construction, in particular how it deals with "fake relations".)

(5) The computation of the cokernel of ev: $M \hookrightarrow M^{\vee \vee}$ relies on [GaS, Lemma 2.1] and can be done very easily in Macaulay 2. The instanton width of $E$ is the dimension of coker(ev) as a $\mathbb{C}$-vector space.

6.2. Computation of instanton heights. We will use the following formula for the height, which is proved in [BGK]:

Theorem 6.3 ([BGK, 2.6]). Let $E$ be a non-split bundle represented in canonical form by $(j, p)$, and let $m>0$ be the smallest exponent of $u$ appearing in $p$. With $\mu=\min \left(m,\left\lfloor\frac{j-2}{k}\right\rfloor\right)$, we have

$$
l\left(R^{1} \pi_{*} E\right)=\mu\left(j-1-k \frac{\mu-1}{2}\right) .
$$

\subsection{Results.}


Lemma 6.5. Let $E_{j}$ be a rank-2 bundle over $Z_{k}$ with $c_{1}=0$ and splitting type $j<k$. Then

$$
\chi^{\mathrm{loc}}\left(E_{j}\right)=j-1 .
$$

Proof. By [Ga2, Theorem 3.3] it follows that if $j<k$ then $E_{j} \cong \mathcal{O}_{Z_{k}}(j) \oplus \mathcal{O}_{Z_{k}}(-j)$. By definition, $\chi^{\text {loc }}\left(E_{j}\right)=\mathbf{w}\left(E_{j}\right)+\mathbf{h}\left(E_{j}\right)$. Direct computation (see [BGK]) then shows that $\mathbf{w}\left(E_{j}\right)=0$ and $\mathbf{h}\left(E_{j}\right)=j-1$.

Proposition 6.7. Let $E$ be a rank-2 reflexive sheaf on $Z_{k}$ with splitting type $\geq k$, then $\chi(x, E) \geq k-1$.

Proof. By semi-continuity of $\chi(x, E)$ on the splitting type, the lowest value of $\chi(x, E)$ must occur for splitting type $k$. By definition,

$$
\chi(x, E) \geq h^{0}\left(X ; R^{1} \pi_{*} E\right),
$$

and now apply the formula (6.4).

Theorem 6.8. The minimal local charge of a nontrivial $S U(2)$-instanton on $Z_{k}$ is $\chi_{k}^{\min }=k-1$. The local moduli space of (unframed) instantons on $Z_{k}$ having fixed local charge $\chi_{k}^{\min }$ has dimension $k-2$.

Proof. By Corollary 5.5, a nontrivial instanton bundle over $Z_{k}$ has splitting type $k n$ for some $n \in \mathbb{Z}, n>0$. Hence, the smallest nontrivial splitting type is exactly $k$, and the generic such instanton corresponds to an element of $\operatorname{Ext}_{Z_{k}}^{1}(\mathcal{O}(k), \mathcal{O}(-k))$ which is nontrivial on the first formal neighbourhood.

The dimension of the local moduli space with fixed $\chi_{k}^{\min }$ is obtained from formula (5.1) setting $j=k$. We compute $\chi$ for the bundle $E$ corresponding to the extension class $[z u] \in \operatorname{Ext}^{1}(\mathcal{O}(k), \mathcal{O}(-k))$.

By Equation (6.4), $\mathbf{h}(E)=k-1$.

To compute $\mathbf{w}(E)=h^{0}\left(X,\left(\pi_{*} E\right)^{\vee \vee} / \pi_{*} E\right)$, we use the method described in Section 6.1: Let $Q$ be the skyscraper sheaf defined by the exact sequence

$$
0 \longrightarrow \pi_{*} E \longrightarrow\left(\pi_{*} E\right)^{\vee \vee} \longrightarrow Q \longrightarrow 0 \text {. }
$$

Then $\mathbf{w}(E)$ equals the dimension of $Q_{x}^{\wedge}$ as a $k_{x}$-module. So, we need to study the map $\pi_{*} E_{x}^{\wedge} \rightarrow\left(\pi_{*} E_{x}^{\wedge}\right)^{\vee \vee}$ and compute the dimension of the cokernel, i.e. we 
need to compute the module structure on $M:=\pi_{*} E_{x}^{\wedge}$ and study the natural map $M \hookrightarrow M^{\vee \vee}$. By the Theorem on Formal Functions (see [Ha1, p. 277]),

$$
M \cong \lim _{n} H^{0}\left(\ell_{n} ;\left.E\right|_{\ell_{n}}\right)
$$

where $\ell_{n}$ is the $n^{\text {th }}$ infinitesimal neighbourhood of $\ell$. Since the extension class has degree 1 in $u$, then for $n \geq 1$,

$$
H^{0}\left(\ell_{n} ;\left.E\right|_{\ell_{n}}\right) \cong H^{0}\left(\ell_{1} ;\left.E\right|_{\ell_{1}}\right)
$$

Therefore, the inverse limit stabilises at 1 , giving $M \cong H^{0}\left(\ell_{1} ;\left.E\right|_{\ell_{1}}\right)$.

To compute the generators of $M$, we write the transition matrix for $E$ explicitly. We set $Z_{k}=U \cup V$, where $U \cong \mathbb{C}^{2} \cong V$, with change of coordinates $U \ni(z, u) \mapsto$ $\left(z^{-1}, z^{k} u\right) \in V$ on $U \cap V \cong \mathbb{C}-\{0\} \times \mathbb{C}$. Then in these coordinate charts, $E$ is given by transition matrix

$$
T=\left(\begin{array}{cc}
z^{k} & z u \\
0 & z^{-k}
\end{array}\right) .
$$

Set $\alpha=\left(\begin{array}{l}u \\ 0\end{array}\right)$ and $\beta_{i}=\left(\begin{array}{c}0 \\ z^{i}\end{array}\right)$ for $i=0, \ldots, k-1, \beta_{k}=\left(\begin{array}{c}-z u \\ z^{k}\end{array}\right)$. Then a presentation for $M$ is given by $M=\left\langle\alpha, \beta_{0}, \ldots, \beta_{k}\right\rangle / R$, where $R$ is the set of relations $\beta_{i} x_{0}-\beta_{i-1} x_{1}=0$ for $i=1, \ldots, k-1$ and $\beta_{k} x_{0}-\beta_{i k-1} x_{1}-\alpha x_{1}=0$. Standard computations (which can be performed either by hand, or with a computer algebra program) then show that the evaluation map $\rho: M \rightarrow M^{\vee \vee}$ is surjective. Hence $\mathbf{w}(E)=0$.

Summing up, $\chi_{k}^{\min }=\chi^{\mathrm{loc}}(E)=\mathbf{w}(E)+\mathbf{h}(E)=0+(k-1)$.

Remark 6.9 (Gaps in local instanton charges). The non-existence of instantons with certain local charges on the spaces $Z_{k}$ when $k>2$ is in stark contrast with what happens in the case $k=1$. In fact, by [BG1, Theorem 0.2], for every non-negative integer $n$ there exist instantons on $Z_{1}$ with local charge $n$. More precisely, by [BG1, Theorem 0.2], for every pair of integers $(w, h)$ satisfying $j-1-e \leq w \leq j(j-1) / 2-j e$ and $1 \leq h \leq j(j+1) / 2$ with $j \geq 0$ and $e \geq 0$ or -1 , there exists a rank- 2 vector bundle $E$ on $Z_{1}$ with splitting type $(j,-j+e)$ having $\mathbf{w}(E)=w$ and $\mathbf{h}(E)=h$. Hence, there are no gaps in local charges for instantons over $Z_{1}$. 


\section{REFERENCES}

[BG1] Ballico, E. and Gasparim, E., Vector bundles on a neighborhood of a curve in a surface and elementary transformations, Forum Math. 15 no. 1, 115-122 (2003).

[BGK] Ballico, E. Gasparim, E. and Köppe, T., Vector bundles near a negative curve: moduli and local Euler characteristic. Preprint.

[Bl] Blache, R., Chern classes and Hirzebruch-Riemann-Roch theorem for coherent sheaves on complex-projective orbifolds with isolated singularities, Math. Z. 222 no. 1, 7-57 (1996).

[Bu] Buchdahl, N. P., Hermitian-Einstein connections and stable vector bundles over compact algebraic surfaces, Math. Ann. 280, 625-648 (1988).

[BHMM] Boyer, C. P., Hurtubise, J. C., Mann, B. M. and Milgram, R. J., The topology of instanton moduli spaces. I. The Atiyah-Jones conjecture. Ann. of Math. (2) 137 no. 3, 561-609 (1993).

[D1] Donaldson, S. K., Instantons and geometric invariant theory, Comm. Math. Phys. 93, 453-460 (1984).

[D2] Donaldson, S. K., Anti-self-dual connections over complex algebraic surfaces and stable vector bundles, Proc. Lond. Math. Soc. (3) 50, 1-26 (1985).

[Ga1] Gasparim, E., The Atiyah-Jones conjecture for rational surfaces. Preprint.

[Ga2] Gasparim, E., Holomorphic bundles on $\mathcal{O}(-k)$ are algebraic, Comm. Algebra 25 no. 10, 3001-3009 (1997).

[GaS] Gasparim, E. and Swanson, I., Computing instanton numbers of curve singularities, J. Symbolic Comput. 40 no. 2, 965-978 (2005)

[GO] Gasparim, E. and Ontaneda, P., Three applications of instanton numbers, Comm. Math. Phys. 270 no.1, 1-12 (2007).

[Ha1] Hartshorne, R., Algebraic geometry. Graduate Texts in Mathematics 52. SpringerVerlag, New York-Heidelberg (1977).

[Ha2] Hartshorne, R., Stable reflexive sheaves, Math. Ann. 254 no. 2, 121-176 (1980).

[HM] Hurtubise, J. and Milgram, R. J., The Atiyah-Jones conjecture for ruled surfaces, J. Reine Angew. Math. 466, 111-143 (1995).

[Kn] King, A., Instantons and holomorphic bundles on the blown-up plane, D. Phil. Thesis, Worcester College, Oxford (1998).

[LB1] LeBrun, C., Counter-examples to the generalized positive action conjecture, Commun. Math. Phys. 118, 591-596 (1988).

[LB2] LeBrun, C., Explicit self-dual metrics on $\mathbb{C} P^{2} \# \cdots \# \mathbb{C} P^{2}$, J. Differential Geom. 34 no. 1, 223-253 (1991).

[LB3] LeBrun, C., Twistors, Kähler manifolds, and bimeromorphic geometry. I. J. Amer. Math. Soc. 5 no. 2, 289-316 (1992).

[LT] Lübke, M. and Teleman, A., The Kobayashi-Hitchin correspondence. World Scientific Publishing Co., Inc., River Edge, NJ (1997). 
[M] Maruyama, M., Elementary transformations in the theory of algebraic vector bundles, Algebraic geometry (La Rábida, 1981), 241-266, Lecture Notes in Math. 961, Springer, Berlin, 1982.

[U] Uhlenbeck, K. Removable singularities in Yang-Mills fields, Comm. Math. Phys. 83 no. 1, 11-29 (1982).

[UY] Uhlenbeck, K. and Yau, S. T., On the existence of Hermitian-Yang-Mills connections in stable vector bundles, Comm. Pure Appl. Math. 39, suppl. S257-S293 (1986).

Elizabeth Gasparim, Thomas Köppe

School of Mathematics, The University of Edinburgh

James Clerk Maxwell Building, The King's Buildings

Mayfield Road, Edinburgh, UK, EH9 3JZ

E-mail: Elizabeth.Gasparim@ed.ac.uk

E-mail: t.koeppe@ed.ac.uk

Pushan Majumdar

Institut für Theoretische Physik

Westfälische Wilhelms-Universität Münster

48149 Münster, Germany

E-mail: pushan@uni-muenster.de 